\title{
Comparative Analysis of Stress Distribution in Composite Resin Brackets with Metal Slot of Permanent Maxillary Central Incisor Using the Finite Element Method:APilot Study
}

\author{
Jae-Jung $\mathrm{Im}^{1}$, Jae-Joon Song ${ }^{1}$, Nan-Hee Kim¹, Jin-Young Heo', \\ Gyu-Un Jung ${ }^{2}$, Kyung-Gyun Hwang ${ }^{3}$, Chang-Joo Park ${ }^{3}$
}

1. Division of Orthodontics, Department of Dentistry, College of Medicine, Hanyang University, Seoul, Korea

2. Division of Periodontology, Department of Dentistry, College of Medicine, Hanyang University, Seoul, Korea

3. Division of Oral and Maxillofacial Surgery, Department of Dentistry, College of Medicine, Hanyang University, Seoul, Korea

\section{Corresponding Author}

\section{Chang-Joo Park, DDS, PhD}

Division of Oral and Maxillofacial Surgery, Department of Dentistry, College of Medicine, Hanyang

University, 17 Haengdang-dong, Seondong-gu, Seoul 133-792, Korea

TEL : +82-2-2290-8646 FAX : +82-2-2290-8673 E-mail : fastchang@hanyang.ac.kr

Received for publication July 26, 2011; Returned after revision September 7, 2011;

Accepted for publication September 16, 2011

\section{- Abstract}

Purpose: For aesthetic reasons, composite resin brackets are widely used. However, related studies are rare. This pilot study sought to compare the stress distributions in two commercially available composite resin brackets with metal slot.

Materials and Methods: Two commercially available resin brackets -- full-metal slot resin bracket (fSRB) and partial-metal slot resin bracket (pSRB) with straight wire appliance dimension of $0.022 \times 0.028$ in -- were selected. In each bracket, 3-dimensional finite element models were constructed, and stress level was evaluated using finite element analysis. By loading the tipping force and torsion moment, which are similar to those applied by the stainless steel rectangular wire $(0.019 \times 0.025-$ in $)$, stress distributions were calculated, and von Mises stress values were obtained.

Results: In pSRB and fSRB, the stress value of the torque moment was much higher than that of the tipping force. The pSRB showed higher stress value than ASRB in both tipping force and torque moment because of the difference in size and configuration of the metal frame inserted into the slot. More stress was also found to be concentrated on the slot area than the wing area in fSRB.

Conclusion: The slot form of fSRB was found to be more resistant to the stress of tipping and torque than the slot form of pSRB. In addition, the slot areas -- rather than the wing areas -- of the bracket showed breakage susceptibility. Therefore, resistance to the torque moment on the slot area should be considered in bracket design.

- Key word : Finite element analysis, Orthodontic bracket, Stress test

\section{- J Kor Dent Sci. 2011; 4(2): 46 - 51}

() This is an open access article distributed under the terms of the Creative Commons Attribution Non-Commercial License (http:// creativecommons.org/licenses/by-nc/3.0) which permits unrestricted non-commercial use, distribution, and reproduction in any medium, provided the original work is properly cited. 


\section{Introduction}

To improve aesthetic appearance, many orthodontic brackets have been developed and released in the market. In the early 1960s, plastic brackets made of polyacrylate resin were introduced. Note, however, that they were not sufficiently strong and were prone to discoloration ${ }^{1,2}$. Composite resin brackets with metal slot were developed since they had the advantage of being neither brittle nor susceptible to fracture and torque loss ${ }^{3)}$. These brackets had stainless-steel metal slot within the plastic bracket, providing less friction to sliding and more resistance to tipping force and torque moment ${ }^{4}$.

Finite element analysis is an effective method of determining stress distribution patterns for the structures of both complex design and known material properties ${ }^{5)}$. According to this method, the actual structure is subdivided into a finite number of independent discrete elements. These elements are then superimposed onto a coordinate grid system to which every node is referenced. Forces are applied on the areas of the model to simulate actual situations. Strains are determined, andstress is evaluated in the stressstrain relationship.

In this pilot study, two commercially available composite resin brackets with metal slot -- a full-metal slot resin bracket (fSRB) and a partial-metal slot resin bracket (pSRB) -of a permanent maxillary central incisor were selected. Though there could be various magnitudes and directions for the applied forces according to the different archwire sizes and compositions, stress distributions by the tipping force and torque moment of stainless steel rectangular wire $(0.019 \times 0.025$-in $)$ were preliminarily analyzed and compared using the finite element method.

\section{Materials and Methods}

Composite resin brackets with metal slot of the permanent

\begin{tabular}{cccc}
\multicolumn{4}{c}{ Table 1. Dimension of brackets } \\
\hline Bracket & Torque (Degrees) & $\begin{array}{c}\text { Angulation } \\
\text { (Degrees) }\end{array}$ & Width (mm) \\
\hline fSRB & 14 & 5 & 3.5 \\
pSRB & 12 & 5 & 3.8 \\
\hline fSRB: full-metal slot resin bracket, pSRB: partial-metal slot resin bracket.
\end{tabular}

Jae-Jung Im, et al: Comparative Analysis of Stress Distribution in Composite Resin Brackets with Metal Slot of Permanent Maxillary Central Incisor Using the Finite Element Method: A Pilot Study. J Kor Dent Sci 2011. maxillary central incisor (dimension: $0.022 \times 0.028$-in.) were a fSRB (Spirit-MB, Ormco, Orange, CA, USA) and a pSRB (Esther II, Tomy, Tokyo, Japan). All brackets had a 0.022in metal slot, and they were pre-adjusted in terms of both torque and angulation to positive rotation of $14^{\circ}$ and $12^{\circ}$ in the palatal root torque (Table 1). fSRB had a metal slot for the base and two vertical walls, whereas pSRB had a metal slot for the base and four vertical wings (Fig. 1). Using the Comet-500 Optical 3D-digitizing System (Steinbichler, Neubeuern, Germany), the force values were measured when force was applied by a $0.019 \times 0.025$-in stainless steel archwire ${ }^{3)}$. The force values were transferred, and the bracket model was constructed with a three-dimensional computer-aided design (CAD) program (AutoCad

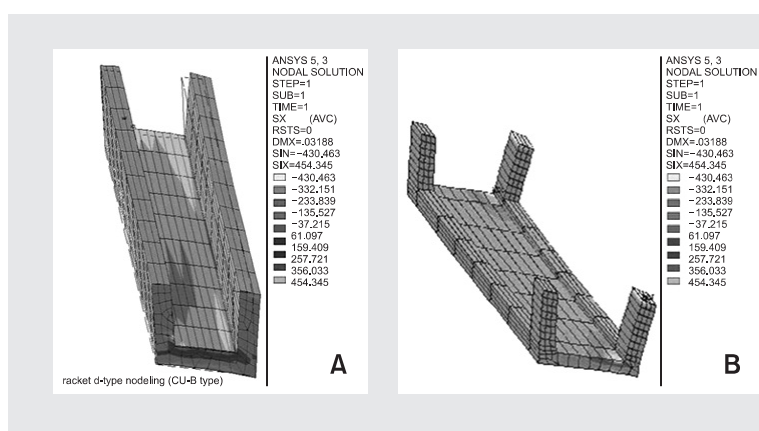

Figure 1. Three-dimensional modeling of metal slots in fSRB (A) and pSRB (B). fSRB: full-metal slot resin bracket, pSRB: partialmetal slot resin bracket.

Jae-Jung Im, et al: Comparative Analysis of Stress Distribution in Composite Resin Brackets with Metal Slot of Permanent Maxillary Central Incisor Using the Finite Element Method: APilot Study. J Kor Dent Sci 2011

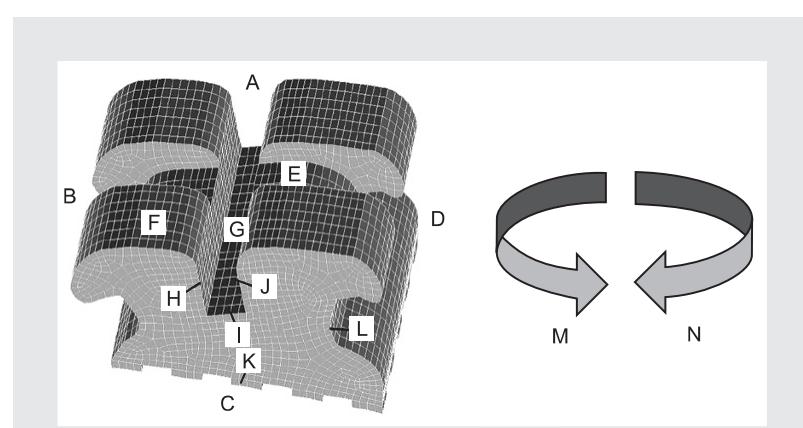

Figure 2. Landmarks on the model bracket (A: mesial, B: incisal, C: distal, D: gingival, E: isthmus, F: wing (frontal), G: metal slot, $\mathrm{H}$ : incisal wall, l: slot base (basal), J: gingival wall, $\mathrm{K}$ : bracket base, L: tying slot), M: counterclockwise rotation, $\mathrm{N}$ : clockwise rotation).

Jae-Jung Im, et al: Comparative Analysis of Stress Distribution in Composite Resin Brackets with Metal Slot of Permanent Maxillary Central Incisor Using the Finite Element Method: A Pilot Study. J Kor Dent Sci 2011. 
2010, Autodesk, San Rafael, CA, USA). Once the closed geometric diagram of the bracket was defined, and all the landmarks of the bracket were designated as in Fig. 2, a mesh was generated with a network of intersecting lines to divide the model into quadrilateral elements by the FE analysis program (Ansys 10.0 Sp, Ansys, Inc., Houston, PA, USA).The physical values of the material properties were set as shown in Table 2 .

Due to the asymmetrical configuration of the brackets in the incisal and gingival direction, different force values were designated. To simulate distal root tipping ${ }^{6}$, 4.27 $\mathrm{N} \cdot \mathrm{mm}$ was applied as tipping force at the mesial-gingival line angle and the disto-incisal line angle. Torque moment of $32.86 \mathrm{~N} \cdot \mathrm{mm}$ was applied at both the incisal and gingival edges of the metal slot in coupled forces.

Table 2. Material properties used in the finito element analysis

\begin{tabular}{ccc}
\hline Material & Elastic modulus $(\mathrm{GPa})$ & Poisson's ratio $(\mathrm{V})$ \\
\hline Polycarbonate & 2.6 & 0.28 \\
Stainless steel & 190 & 0.3 \\
\hline
\end{tabular}

Jae-Jung Im, et al: Comparative Analysis of Stress Distribution in Composite Resin Brackets with Metal Slot of Permanent Maxillary Central Incisor Using the Finite Element Method: A Pilot Study. J Kor Dent Sci 2011.

\begin{tabular}{ccc}
\multicolumn{3}{c}{ Table 3. Maximum stress at tipping forces } \\
\hline Bracket & X-direction (MPa) & Y-direction (MPa) \\
\hline fSRB & 279 & 151 \\
pSRB & 125 & 72 \\
\hline fSRB: full-metal slot resin bracket, pSRB: partial-metal slot resin bracket.
\end{tabular}

Jae-Jung Im, et al: Comparative Analysis of Stress Distribution in Composite Resin Brackets with Metal Slot of Permanent Maxillary Central Incisor Using the Finite Element Method: A Pilot Study. J Kor Dent Sci 2011.

\section{Results}

\section{Tipping forces}

The maximum stress value at one point of the entire node system for fSRB was $279 \mathrm{MPa}$ in the $\mathrm{x}$-direction and 151 $\mathrm{MPa}$ in the y-direction; it was $125 \mathrm{MPa}$ in the $\mathrm{x}$-direction and $72 \mathrm{MPa}$ in the y-direction for pSRB (Table 3). Although the maximum stress value at one point was higher in ISRB than in pSRB, the sum of stress generated by the applied force on entire node system was higher in pSRB. The major stresses concentrated along two lines: at the mesial-gingival line angle and the disto-incisal line angle (Fig. 3).

\section{Torque moments}

When counterclockwise rotation (palatal crown torque: Figs. 2, 4A, 4C) was made on the torque moment, the maximum stresses in the $\mathrm{x}$ - and $\mathrm{y}$-directions were $470 \mathrm{MPa}$ and $456 \mathrm{MPa}$ in $\mathrm{fSRB}$, respectively, and $858 \mathrm{MPa}$ and 859 $\mathrm{MPa}$ in $\mathrm{pSRB}$, respectively. When clockwise rotation (buccal crown torque: Figs. 2, 4B, 4D) was made on the torque moment, the maximum stresses in the $\mathrm{x}$ - and $\mathrm{y}$ directions were $622 \mathrm{MPa}$ and $567 \mathrm{MPa}$ in fSRB, respec-

\section{\begin{tabular}{ccccc} 
& \multicolumn{3}{c}{ Table 4. Maximum stress at torque moments } \\
\hline \multirow{3}{*}{ Bracket } & \multicolumn{2}{c}{ Counterclockwise rotation } & \multicolumn{2}{c}{ Clockwise rotation } \\
& \multicolumn{2}{c}{ (MPa) } & \multicolumn{2}{c}{ (MPa) } \\
\cline { 2 - 5 } & X-direction & Y-direction & X-direction & Y-direction \\
\hline fSRB & 470 & 456 & 622 & 567 \\
pSRB & 858 & 859 & 1,152 & 1,410 \\
\hline fSRB: full-metal slot resin bracket, pSRB: partial-metal slot resin bracket.
\end{tabular} \\ Jae-Jung Im, et al: Comparative Analysis of Stress Distribution in Composite Resin Brackets with Metal Slot of Permanent Maxillary Central Incisor Using the Finite Element Method: A Pilot Study. J Kor Dent Sci 2011.}

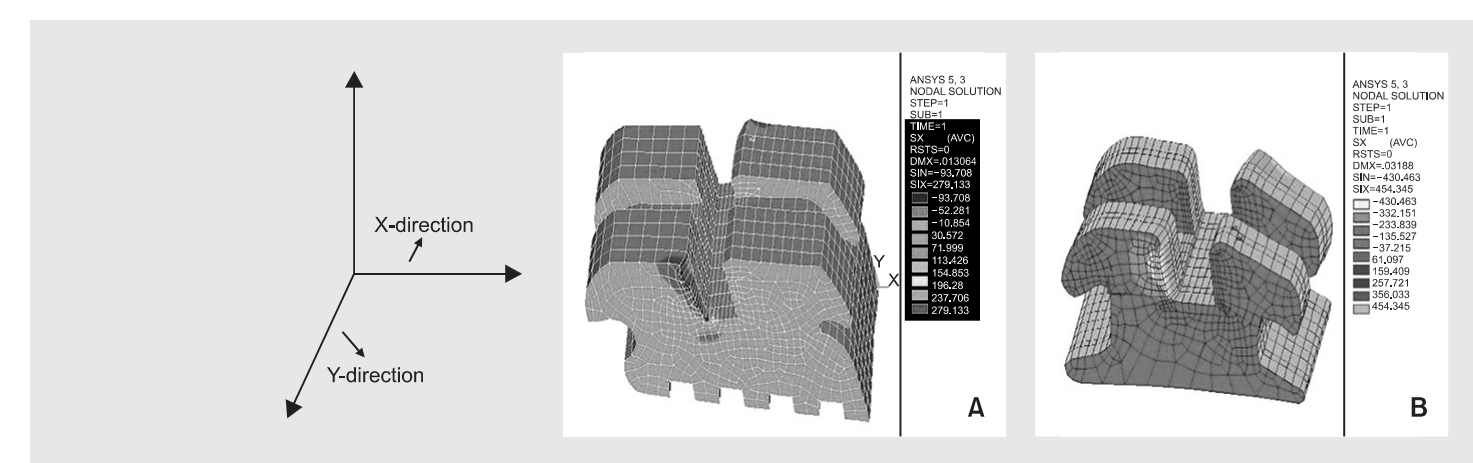

Figure 3. Stress distribution pattern with tipping force in fSRB (A) and pSRB (B). fSRB: full-metal slot resin bracket, pSRB: partial-metal slot resin bracket.

Jae-Jung Im, et al: Comparative Analysis of Stress Distribution in Composite Resin Brackets with Metal Slot of Permanent Maxillary Central Incisor Using the Finite Element Method: A Pilot Study. J Kor Dent Sci 2011. 


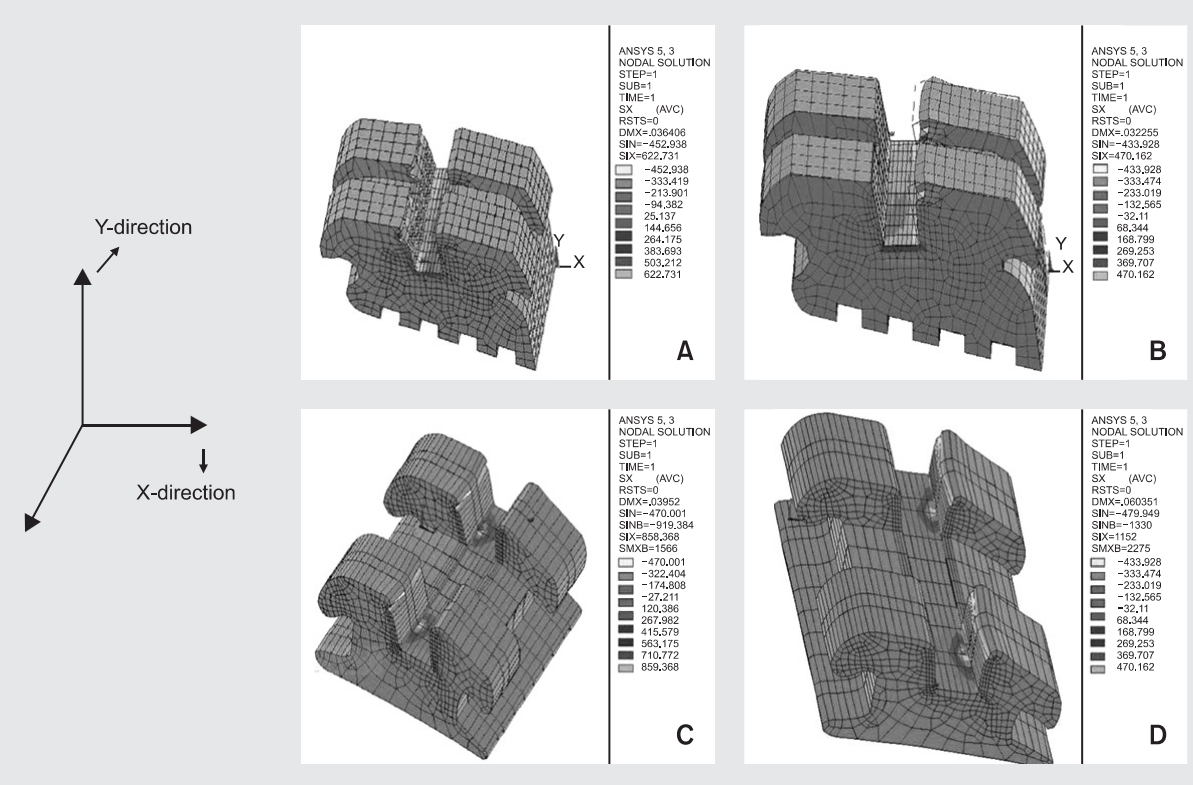

Figure 4. Stress distribution pattern with torquemoment in fSRB (A: counterclockwise rotation, B: clockwise rotation) and in pSRB (C: counterclockwise rotation, D: clockwise rotation). fSRB: full-metal slot resin bracket, pSRB: partial-metal slot resin bracket.

Jae-Jung Im, et al: Comparative Analysis of Stress Distribution in Composite Resin Brackets with Metal Slot of Permanent Maxillary Central Incisor Using the Finite Element Method: A Pilot Study. J Kor Dent Sci 2011.

tively, and 1,152 MPa and 1,410 MPa in pSRB, respectively (Table 4). Higher stress values were shown with the torquemoment than the tipping force, with the difference in rotational direction affecting the stress value. The major stresses on the brackets were concentrated along two lines: at the fronto-gingival line angle and the baso-incisal line angle (Fig. 4D).

\section{Discussion}

There are many aesthetic brackets such as ceramic, composite resin, and lingual brackets. Many research studies have been conducted on ceramic brackets ${ }^{7-11)}$. Note, however, that studies on the composite resin bracket were relatively rare. In this study, the stress distributions of two composite resin brackets with metal slot -- fSRB and pSRB -were evaluated by the finite element method.

For the tipping force on the fSRB and pSRB of the permanent maxillary central incisor, stresses were distributed in a similar manner and concentrated along two lines: at the mesial-gingival line angle and the disto-incisal line angle. Previous studies also showed that maximum stress was concentrated on the mesial-gingival and disto-incisal wing areas ${ }^{12)}$. Since the stress in the $\mathrm{x}$-direction was found to be higher than that in the y-direction because of the direction of tipping force, special considerations for this direction should be taken in bracket designs. Although found to show higher stress, fSRB actually recorded lower observed stress. The fSRB came into contact with a more extensive area of polycarbonate than $\mathrm{pSRB}$; therefore, the effect of polycarbonate deformation was higher in fSRB than pSRB. This is explained by the low elastic modulus of polycarbonate. When we consider the equation for stress: $\sigma=E \varepsilon(\sigma$ : stress, E: elastic modulus, $\varepsilon$ : strain), if the elastic modulus of the material is low, even though the same amount of strain occurs, the stress level will be low. Since stress was higher in pSRB than fSRB, the stress was more widely distributed in $\mathrm{pSRB}$ than fSRB. Although this does not seem to be enough to affect the destruction of pSRB, wing deformation by the tipping force -- which was related to the design of the bracket-- was observed. This may be remedied by the creation of a reinforced metal slot inserted into the bracket ${ }^{13)}$. Augmentation by the metal slot appears to show stability in the bracket due to action on the interface of the wire slot in the bracket.

The torque moment on the fSRB and pSRB of the permanent maxillary central incisor was loaded on a greater number of nodes than the tipping force as shown in Figs. 3 and 
4, and the torque moment was nine times higher than the tipping force. It means that it is the torque force rather than the tipping force that causes the breakage. The torque moment of the counter clockwise rotation (palatal crown torque) was higher than that of the clockwise rotation (buccal crown torque). Based on the asymmetrical geometry of the bracket configuration, this could be explained by the difference in base thickness, i.e., thinner in the incisor wing area than in the gingival area.

Comparing the two brackets in both tipping force and torque moment, the higher stress in pSRB than in fSRB of the permanent maxillary central incisor was due to the difference in size and configuration in the metal frame inserted into the slot. Because the metal slot of pSRB was not completely covered, the stress of pSRB in torque moment was found to be higher than that of fSRB. Therefore, pSRB is assumed to be more susceptible to deformation than fSRB. The pattern of stress distributions appeared to be similar in both brackets. Note, however, that the slot area of the bracket had greater susceptibility to breakage than the wing area because of the two or three times' higher stress level in the torque force compared to the tipping force. Stresses were widely distributed along the base of the slot and concentrated at the fronto-gingival line angle and the baso-incisal line ${ }^{5,12)}$. To obtain greater strength for the bracket, in bracket design, the composite resin bracket should have a full metal slot that completely covers the entire base and vertical wall since complex stress distribution -- which causes deforma-

\section{References}

\footnotetext{
1. Aird JC, Durning P. Fracture of polycarbonate edgewise brackets: a clinical and SEM study. Br J Orthod. 1987; 14: 191-5.

2. Dobrin RJ, Kamel IL, Musich DR. Load-deformation characteristics of polycarbonate orthodontic brackets. Am J Orthod. 1975; 67: 24-33.

3. Morina E, Eliades T, Pandis N, Jäger A, Bourauel C. Torque expression of self-ligating brackets compared with conventional metallic, ceramic, and plastic brackets. Eur J Orthod. 2008; 30: 233-8.

4. Feldner JC, Sarkar NK, Sheridan JJ, Lancaster DM. In vitro torquedeformation characteristics of orthodontic polycarbonate brackets. Am J Orthod Dentofacial Orthop. 1994; 106: 265-72.

5. Hioki M, Shin-Ya A, Nakahara R, Vallittu PK, Nakasone Y, Shin-Ya A. Shear bond strength and FEM of a resin-modified glass ionomer cement--effects of tooth enamel shape and orthodontic bracket base configuration. Dent Mater J. 2007; 26: 700-7.

6. Ghosh J, Nanda RS, Duncanson MG Jr, Currier GF. Ceramic bracket
}

tion -- exists in the wing area where polycarbonate comes into contact with the metal slot ${ }^{2}$.

In this pilot study, there were limitations in the directions and magnitudes of applied forces attributed to the different archwire sizes and compositions. In addition, tipping force and torque moment were considered separately, yet these forces are loaded simultaneously and complicatedly in actual clinical setting. Therefore, these factors should be taken into consideration for further clinical interpretation and application.

\section{Conclusion}

In tipping and torque, the stress distribution of pSRB was higher than that of fSRB.These results depended almost entirely on the slot form inserted into the bracket and the bracket configuration related to such. The slot area of the bracket had greater susceptibility to breakage than the wing area because of the two or three times' higher stress level in the torque force compared to the tipping force. Therefore, resistance to the torque force on the slot area is recommended for future bracket designs. The difference in stress value between the clockwise and counterclockwise rotations is attributed to the asymmetrical configuration of the bracket and the location of the node according to the rotation direction. Composite resin brackets with full metal slot might be more resistant to stresses of tipping and torque than those with partial metal slot. design: an analysis using the finite element method. Am J Orthod Dentofacial Orthop. 1995; 108: 575-82

7. Reitan K. Some factors determining the evaluation of forces in orthodontic. Am J Orthod. 1957; 43: 32-45.

8. Scott GE Jr. Fracture toughness and surface cracks--the key to understanding ceramic brackets. Angle Orthod. 1988; 58: 5-8.

9. Holt MH, Nanda RS, Duncanson MG Jr. Fracture resistance of ceramic brackets during arch wire torsion. Am J Orthod Dentofacial Orthop. 1991; 99: 287-93

10. Tanne K, Matsubara S, Shibaguchi T, Sakuda M. Wire friction from ceramic brackets during simulated canine retraction. Angle Orthod. 1991; 61: 285-90.

11. Flores DA, Choi LK, Caruso JM, Tomlinson JL, Scott GE, Jeiroudi MT. Deformation of metal brackets: a comparative study. Angle Orthod. 1994; 64: 283-90. 


\section{References}

12. Rhodes RK, Duncanson MG Jr, Nanda RS, Currier GF. Fracture strengths of ceramic brackets subjected to mesial-distal archwire tipping forces. Angle Orthod. 1992; 62: 67-76.
13. Jones SP, Amoah KG. Static frictional resistances of polycrystalline ceramic brackets with conventional slots, glazed slots and metal slot inserts. Aust Orthod J. 2007; 23: 36-40. 